\title{
Arrangement of Guldin Second Theorem and Its Application in Definite Integral Calculation
}

\author{
Yong Huang \\ School of Mathematics and Statistics, Zhaotong University, Zhaotong Yunnan, 657000, China
}

Key words: Guldin second theorem, Definite integral calculation, Application.

\begin{abstract}
Guldin theorem is an important theorem, but is scarcely mentioned in the Higher Mathematics for mathematics majors and other majors, except that is its roughly mentioned in the Higher Mathematics for physics major. However, Guldin theorem is useful for simplifying mathematical calculation both in elementary geometry and of hydraulic pressure and work in physics according to its "idea of centre of gravity", to convert calculus operation into elementary operation. It is of special value to applying higher mathematics in engineering technology and production.
\end{abstract}

\section{Propaedeutics and Guldin Theorem}

Lemma $^{[1]}$ If there are $n$ material points in a plane: $M_{1}\left(x_{1}, y_{1}\right), M_{2}\left(x_{2}, y_{2}\right), \cdots, M_{n}\left(x_{n}, y_{n}\right)$, of which the masses are $m_{1}, m_{2} \cdots, m_{n}$ respectively, then their barycentric coordinates $G(\bar{x}, \bar{y})$ are

$$
\left\{\begin{array}{c}
\bar{x}=\frac{M_{y}}{M}=\frac{\sum_{i=1}^{n} x_{i} m_{i}}{\sum_{i=1}^{n} m_{i}}=\frac{x_{1} m_{1}+x_{2} m_{2}+\cdots+x_{n} m_{n}}{m_{1}+m_{2}+\cdots+m_{n}} \\
\bar{y}=\frac{M_{x}}{M}=\frac{\sum_{i=1}^{n} y_{i} m_{i}}{\sum_{i=1}^{n} m_{i}}=\frac{y_{1} m_{1}+y_{2} m_{2}+\cdots+y_{n} m_{n}}{m_{1}+m_{2}+\cdots+m_{n}}
\end{array}\right.
$$

Or

$$
M_{y}=\bar{x} M \quad M_{x}=\bar{y} M
$$

Wherein, $M_{x}, M_{y}$ are the static torques of $\mathrm{n}$ material points relative to x and y axes respectively (the static torques of material point of which the mass is $m$ relative to $\mathrm{x}$ and $\mathrm{y}$ axes equal to the product of the mass $\mathrm{m}$ multiplying the distances of the point to $\mathrm{x}$ and $\mathrm{y}$ axes, namely the product of $\mathrm{m}$ multiplying x and y); $M$ refers to the sum of the masses of $n$ material points. If all the masses of the material point group concentrate at the centre of gravity, the static torque of the material point group relative to an axis equals to that of the material point group relative to the same axis.

Deduction 1 Suppose a smooth curved section $y=f(x), x \in[a, b]$, its linear density is a constant $\mu$, then its barycentric coordinates $G(\bar{x}, \bar{y})$ are 


$$
\left\{\begin{array}{c}
\bar{x}=\frac{M_{y}}{M}=\frac{\int_{a}^{b} x \sqrt{1+f^{\prime 2}(x)} d x}{\int_{a}^{b} \sqrt{1+f^{\prime 2}(x)} d x} \\
\bar{y}=\frac{M_{x}}{M}=\frac{\int_{a}^{b} f(x) \sqrt{1+f^{\prime 2}(x)} d x}{\int_{a}^{b} \sqrt{1+f^{\prime 2}(x)} d x}
\end{array}\right.
$$

Deduction 2 Suppose a trapezoid with curve side is formed by a continuous curve $y=f(x)$ and a straight line $x=a, x=b, y=0(a<b)$, and its area density is a constant $\mu$, then its barycentric coordinates $G(\bar{x}, \bar{y})$ are $\check{\mathrm{S}}$

$$
\left\{\begin{array}{l}
\bar{x}=\frac{M_{y}}{M}=\frac{\int_{a}^{b} x f(x) d x}{\int_{a}^{b} f(x) d x} \\
\bar{y}=\frac{M_{x}}{M}=\frac{\int_{a}^{b} f^{2}(x) d x}{2 \int_{a}^{b} f(x) d x}
\end{array}\right.
$$

Deduction 3 Suppose a planar graph is formed by continuous curves $y=f(x), y=g(x)(f(x) \leq g(x))$ and a straight line $x=a, x=b(a<b)$, and its area density is a constant $\mu$, then its barycentric coordinates $G(\bar{x}, \bar{y})$ are

$$
\left\{\begin{array}{c}
\bar{x}=\frac{M_{y}}{M}=\frac{\int_{a}^{b} x[g(x)-f(x)] d x}{\int_{a}^{b}[g(x)-f(x)] d x} \\
\bar{y}=\frac{M_{x}}{M}=\frac{\int_{a}^{b}\left[g^{2}(x)-f^{2}(x)\right] d x}{2 \int_{a}^{b}[g(x)-f(x)] d x}
\end{array}\right.
$$

The following is the proof of Deduction 2.

Proof: Suppose a planar sheet (a trapezoid with curve side) is formed by a curve $y=f(x)$ and a straight line $x=a, x=b, y=0$, and its area density is a constant $\mu$.

Partition the closed interval $[a, b]$ into $n$ short intervals with any group of points of division, then the whole planar sheet is divided into $n$ narrow strips, wherein the mass of the narrow strip of which the width is $d x$ in $\forall x \in[a, b]$ can be approximately replaced with a material point of which the coordinates are $\left(x, \frac{1}{2} f(x)\right)$. Since the mass of the narrow strip is about $d m=\mu f(x) d x$, then the static torques of the material point $\left(x, \frac{1}{2} f(x)\right)$ relative to $\mathrm{y}$ and $\mathrm{x}$ axes are

$$
m_{y}=x d m=\mu x f(x) d x, \quad m_{x}=\frac{1}{2} f(x) d m=\frac{1}{2} \mu f^{2}(x) d x
$$

Then the static torques of the planar sheet relative to $\mathrm{y}$ and $\mathrm{x}$ axes are

$$
M_{y}=\mu \int_{a}^{b} x f(x) d x, \quad M_{x}=\frac{\mu}{2} \int_{a}^{b} f^{2}(x) d x .
$$



is

Since the total mass of the planar sheet is $M=\mu S=\mu \int_{a}^{b} f(x) d x$, the barycenter of the planar sheet

$$
\left\{\begin{array}{l}
\bar{x}=\frac{M_{y}}{M}=\frac{\int_{a}^{b} x f(x) d x}{\int_{a}^{b} f(x) d x} \\
\bar{y}=\frac{M_{x}}{M}=\frac{\int_{a}^{b} f^{2}(x) d x}{2 \int_{a}^{b} f(x) d x}
\end{array}\right.
$$

Theorem 1 (Guldin First Theorem) A planar curve rotates around an axis that doesn't intersect therewith (its periphery is ok), and the lateral area of the generated solid of rotation equals to the product of the circumference of the circle formed by the barycenter of the curve rotating around the same axis multiplying the arc length of the curve.

Theorem 2 (Guldin Second Theorem) The volume of the solid produced by a planar figure rotating around an axis that doesn't intersect therewith (its periphery is ok) equals to the product of the area of the planar figure multiplying the circumference of its barycenter rotating around an axis.

The following is the proof of Guldin Second Theorem.

Proof: Without loss of generality, suppose a planar figure A is formed by curves $y=f(x), y=g(x)$ and straight lines $x=a, x=b$ (see figure), its area is S, and its areal density is a constant $\mu$, then the mass of the planar figure is $M=\mu S$; suppose $f(x) \leq g(x), \quad f(x) \geq 0, g(x) \geq 0$,.

Then, the static torques of the planar figure relative to $x$ and $y$ axes are

$$
\begin{aligned}
& M_{x}=\frac{\mu}{2} \int_{a}^{b}\left[g^{2}(x)-f^{2}(x)\right] d x \\
& M_{y}=\mu \int_{a}^{b} x[g(x)-f(x)] d x
\end{aligned}
$$

Then the y coordinate of the barycenter of the

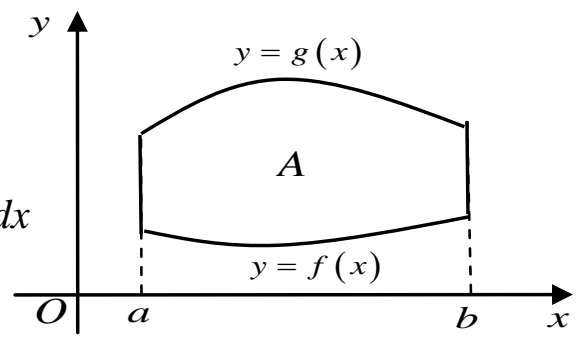

figure is

$$
\bar{y}=\frac{M_{x}}{M}=\frac{\int_{a}^{b}\left[g^{2}(x)-f^{2}(x)\right] d x}{2 S}
$$

Since the volume of the solid produced by the figure rotating a circle around $\mathrm{x}$ axis is $V_{x}=\pi \int_{a}^{b}\left[g^{2}(x)-f^{2}(x)\right] d x$

Then

$$
\pi S \bar{y}=\frac{1}{2} V_{x}
$$

\section{Namely}

It is proved by the same methods that

$$
V_{x}=S \cdot 2 \pi \bar{y}
$$$$
V_{y}=S \cdot 2 \pi \bar{x}
$$

In fact, $\forall x \in[a, b]$, the infinitesimal of the volume of the solid of rotation produced by a small area of which the width is $\mathrm{d}^{x}$ and the height is $f(x)$ is

$$
d V=2 \pi x f(x) d x \quad\left(\Delta V=\pi\left[(x+d x)^{2}-x^{2}\right] f(x)\right)
$$


Then, the volume of the solid of rotation produced by a figure formed by $y=f(x), \quad x=a, x=b$ and $\mathrm{x}$ axis is

Likewise, the volume of the solid of rotation produced by a figure formed by $y=g(x), x=a, x=b$ and $x$ axis is

$$
2 \pi \int_{a}^{b} x g(x) d x
$$

Then, the volume of the solid of rotation produced by the original planar figure rotating around y axis is

$$
V_{y}=2 \pi \int_{a}^{b} x g(x) d x-2 \pi \int_{a}^{b} x f(x) d x=2 \pi \int_{a}^{b} x[g(x)-f(x)] d x
$$

Since the static torque of the figure relative to y axis is $M_{y}=\mu \int_{a}^{b} x[g(x)-f(x)] d x, M=\mu S$

Thus, the abscissa of the barycenter of the figure is $\begin{gathered}\bar{x}=\frac{M_{y}}{M}=\frac{\int_{a}^{b} x[g(x)-f(x)] d x}{S}=\frac{\frac{1}{2 \pi} V_{y}}{S} \\ \text { Namely }\end{gathered} \quad V_{y}=S \cdot 2 \pi \bar{x}$

\section{Barycenter Thought of Guldin Theorem}

The Guldin First Theorem gives a method of calculating the area of the surface of revolution produced by a planar curve $\Gamma$ rotating around an axis $L$ that doesn't intersect therewith (the extreme point(s) of the curve $\Gamma$ can intersect with $L$ ). In the event that it is easy to calculate the distance between the barycenter of the curve and the axis and the length of the curve, it is convenient to solve the area of the surface of revolution by the Guldin First Theorem.

The Guldin Second Theorem gives a method of calculating the volume of the solid of rotation produced by a planar figure $\Sigma$ rotating a circle around an axis L that doesn't intersect therewith (the periphery of the curve ${ }^{\Sigma}$ can intersect with L). likewise, in the event that it is easy to calculate the distance between the barycenter of the plane and the axis and the area of the plane, it is convenient to solve the volume of the solid of rotation by the Guldin Second Theorem.

There are two determining factors in calculating the lateral area and volume of the solid of rotation, namely the size of the curve $\Gamma$ or the planar figure $\Sigma$ before rotating, and the distance of rotating of each point (namely arc length). Since the radius of gyration varies from point to point, it needs to choose a representative point on the curve or planar figure, of which the movement distance should be the average value of the movement distances of all points. Naturally, this is the characteristic of a barycenter. In the Guldin theorems, this important concept in physics is utilized, all "masses" of a geometry are concentrated, a geometry is taken as a material point group, and all masses of the group all concentrate on the barycenter, then the static torque of the geometry relative to an axis (product of the mass $m$ of material point multiplying the distance between the point and the axis) equals to that of the material point group relative to the same axis. The barycenter thought has been extended to curve and plane. Thus, the problem of solving the barycenter is converted to solving the static torque [2].

\section{Application of Guldin Theorem}

\section{Geometry-related Calculations}

Barycenter 
Eg. 1 Solve the barycenter coordinates of an area of $\frac{x^{2}}{a^{2}}+\frac{y^{2}}{b^{2}} \leq 1 \quad(0 \leq x \leq a, 0 \leq y \leq b)$.

Solution: The volume of the solid of rotation produced by the given area rotating around $x$ axis is $\frac{1}{2}$ of that of an ellipsoid $\frac{x^{2}}{a^{2}}+\frac{y^{2}}{b^{2}}+\frac{z^{2}}{b^{2}}=1$, namely $\frac{2}{3} \pi a b^{2}$, and the given area is $S=\frac{a b \pi}{4}$. According to the Guldin Second Theorem,

$$
\begin{array}{r}
\frac{2}{3} \pi a b^{2}=\frac{a b \pi}{4} \cdot 2 \pi \bar{y} \\
\text { Namely } \quad \bar{y}=\frac{4 b}{3 \pi}
\end{array}
$$

Likewise, the volume of the solid of rotation produced by the given area rotating around $y$ axis is $\frac{1}{2}$ of that of the ellipsoid $\frac{x^{2}}{a^{2}}+\frac{y^{2}}{b^{2}}+\frac{z^{2}}{b^{2}}=1$, namely $\frac{2}{3} \pi a^{2} b$. According to the Guldin Second Theorem,

$$
\frac{2}{3} \pi a^{2} b=\frac{a b \pi}{4} \cdot 2 \pi \bar{x}
$$

Namely $\bar{x}=\frac{4 a}{3 \pi}$, then the desired barycenter coordinates are $\left(\frac{4 a}{3 \pi}, \frac{4 b}{3 \pi}\right)$. Lateral Area and Volume

Eg. 2 Calculate the lateral area and volume of the solid of rotation produced by a circle $x^{2}+(y-b)^{2}=a^{2}(b \geq a)$ rotating around $x$.

Solution: Obviously, the barycenter coordinates of the circle are $\bar{x}=0, \bar{y}=b$. According to the Guldin First Theorem,

The lateral area of the solid of rotation is $S=2 \pi b \cdot 2 \pi a=4 \pi^{2} a b$

Since the area of the circle is $S_{1}=\pi a^{2}$. According to the Guldin Second Theorem, the volume of the solid of rotation is

$$
V=S_{1} \cdot 2 \pi \bar{y}=\pi a^{2} \cdot 2 \pi b=2 \pi^{2} a^{2} b
$$

In the event that it is easy to solve the area $\mathrm{S}$ of a planar figure, and its barycenter is known, it is convenient to calculate the volumes of the solid of rotation produced by it rotating around the coordinate axes according to Guldin Theorems; conversely, if the volumes of the solid of rotation produced by a planar figure rotating around the coordinate axes are known, it is also able to calculate the barycenter coordinates of the figure quickly according to Guldin Theorems. Thus, Guldin Theorems are the bridge linking area, volume and barycenter coordinates [3].

\section{Calculation of Pressure from Liquid on One Side of A Sheet Placed Vertical to the Liquid Level}

Suppose the specific gravity of liquid is $r$, the static pressure of liquid at the same depth, of which the value equals to the product of the specific gravity of liquid multiplying the depth. In the event that $\Delta x$ is very small, the static pressure on the narrow strip from $x$ to $x+\Delta x$ of the sheet is

$$
\Delta p \approx d p=\operatorname{rxf}(x) d x .
$$

The hydraulic pressure $\mathrm{p}$ on the planar sheet is

$$
p=\int_{a}^{b} d p=\int_{a}^{b} r x f(x) d x=r \cdot A \cdot \frac{\int_{a}^{b} x f(x) d x}{A}=r \cdot A \cdot \frac{\int_{a}^{b} x f(x) d x}{\int_{a}^{b} f(x) d x}=r \cdot A \cdot h
$$

Where, A refers to the area of the planar sheet, and $\mathrm{h}$ to the distance of the barycenter of the planar sheet to the liquid level[4]. 
Eg. 3 Suppose an isosceles triangle sheet of which the bottom is $8 \mathrm{~cm}$ and the height is $6 \mathrm{~cm}$ sinks in the water vertically with the top above and $3 \mathrm{~cm}$ away from the water level and the bottom below and in parallel with the water level, please solve the pressure on side of the triangle sheet.

Solution: Since the density of water is $10^{3} \mathrm{~kg} / \mathrm{m}^{3}$, the area of the isosceles triangle sheet is $\frac{1}{2} \cdot 8 \cdot 6 \mathrm{~cm}^{2}$, and the distance from the barycenter to the water level is $3+\frac{2}{3} \cdot 6 \mathrm{~cm}$, then the pressure on side of the triangle sheet is

$$
p=r \cdot A \cdot h=9.8 \times 10^{3} \times 24 \times 7=164.64(N)
$$

\section{Work-related Calculations}

Suppose the specific gravity of liquid is $\mathrm{r}$, the volume is $\mathrm{V}$, and the distance from the barycenter to the liquid level is h, according to the deducing method of Paragraph 3.2, the work required to evacuate liquid is

$$
W=\int_{a}^{b} F(x) d x=r \cdot V \cdot h
$$

Eg. 4 Suppose there is a cylindrical pond of which the depth is $18 \mathrm{~m}$, and the diameter is $20 \mathrm{~m}$, and it is planned to evacuate water from the $16 \mathrm{~m}$-deep pond with a pump, please solve the work the pump makes.

Solution: The density of water is $10^{3} \mathrm{~kg} / \mathrm{m}^{3}$, the volume of water in the pond is $\pi \cdot 10^{2} \cdot 16 \mathrm{~m}^{3}$, and the distance from the barycenter to the surface of the pond is $\left(18-16+\frac{16}{2}\right) m$, then the work to be made by the pump is

$$
W=9.8 \times 10^{3} \times 1600 \pi \times 10 \approx 1.57 \times 10^{8} \pi(J)
$$

\section{Considerations on Application of Guldin Theorems}

Guldin Theorems are mainly used to simply calculations of definite integral calculation of geometry, hydraulic pressure, and work, and so on. But it must be noted that only in the event that it is easy to solve the position of the barycenter and area of the geometry, the Guldin Theorems work. Take the example of the 2nd example above. If $|b|<a$, the solid of rotation produced by the given circle $x^{2}+(y-b)^{2}=a^{2}$ rotating around $x$ axis is a solid of rotation produced by the major arch rotating (the solid of rotation produced by the inferior arch is contained in that produced by the major arch). Since it is complicated to calculate the barycenter of an arch, the Guldin Theorems are not used [5].

Besides, the Guldin Theorems also can be applied in engineering technology and other fields, such as the calculation of mass of parts of a solid of rotation of which the shape is irregular in the process of fan design. The calculative process is as below. The volume of the parts of the solid of rotation will be solved first. If the density of the parts is given, then the mass of the parts can be solved. About such questions, the discussions are omitted here.

\section{References}

[1] Higher Mathematics Teaching and Research Office of College of Mathematics S.C.U., Higher Mathematics (Physics Major) Volume I , Beijing, Higher Education Press, 1987:350-353.

[2] Fang Hongzhu, Cai Chengwen. Utilize "Barycenter” to Simplify Calculation, Journal of Nanjing Vocational Institute of Industry Technology, 2005, 9: 93-95.

[3] Gao Shuzhu, Xu Chunhua. Reconsideration on Volume of Solid of Rotation, Shuxue Tongbao,2005, (1): 54 -57. 
[4] B. II. Demidovich. Mathematics Analysis Exercise Book (Volume III), Jinan: Shandong Science Publisher, 1981.

[5] Chen Zhihua. Research on Calculation of Volume of Solid of Rotation Produced by A Plane Rotating Around A Fixed Straight Line, Journal of Science of Teachers College and University, 2008, 5:38-40. 